\title{
Design Considerations for Stability of Liquid Payload Projectiles
}

\author{
Jonathan Rogers* \\ Texas A\&M University, College Station, Texas 77843 \\ Mark Costello \\ Georgia Institute of Technology, Atlanta, Georgia 30332 \\ and \\ Gene Cooper辛 \\ U.S. Army Research Laboratory, Aberdeen Proving Ground, Maryland 21005
}

DOI: $10.2514 / 1 . A 32292$

\begin{abstract}
It is well known that projectiles equipped with liquid payloads experience large destabilizing moments induced by internal motion of the fluid. For some configurations, these moments may lead to catastrophic flight instabilities. This paper explores how payload geometry, fluid spin-up rate, and the magnitude of launch perturbations affect the flight trajectories of projectiles with a liquid payload, including projectile instability. A dynamic simulation model is used in which a projectile rigid-body dynamics representation incorporates liquid moments generated from linearized Navier-Stokes predictions. A novel feature of this simulation is its use of linear filtering within the model, which determines fast- and slow-mode angle-of-attack contributions at each time step to be used in computation of liquid moments. Example cases and Monte Carlo simulations demonstrate how design factors and launch perturbations affect the projectile's tendency to exhibit flight instability. The paper concludes with a summary of advantageous design characteristics that are likely to mitigate the effects of destabilizing liquid moments.
\end{abstract}

\begin{tabular}{|c|c|}
\hline & Nomenclature \\
\hline AR & $=$ liquid cavity equivalent cylinder aspect ratio \\
\hline$C_{\mathrm{LSM}}$ & liquid side moment coefficient \\
\hline$C_{\mathrm{LIM}}$ & $=$ liquid overturning moment coefficient \\
\hline$C_{\mathrm{LRM}}$ & $=$ liquid roll moment coefficient \\
\hline$C_{i}$ & $=$ various aerodynamic coefficients \\
\hline$c_{v}$ & $=$ liquid cylinder viscous roll bearing coefficient \\
\hline$D$ & $=$ projectile reference diameter \\
\hline$g$ & $=9.8 \mathrm{~m} / \mathrm{s}^{2}$, gravitational constant \\
\hline$I$ & $\begin{array}{l}=\text { total moment of inertia matrix of projectile and } \\
\text { liquid payload about the composite mass center }\end{array}$ \\
\hline $\mathbf{I}_{I}, \mathbf{J}_{I}, \mathbf{K}_{I}$ & $=$ inertial reference frame unit vectors \\
\hline $\mathbf{I}_{P}, \mathbf{J}_{P}, \mathbf{K}_{P}$ & $=$ projectile reference frame unit vectors \\
\hline$L, M, N$ & $\begin{array}{l}=\text { total moment applied to the projectile about the } \\
\text { total mass center }\end{array}$ \\
\hline$m$ & $=$ total mass of the projectile and liquid payload \\
\hline$m_{L}$ & $=$ mass of the liquid payload \\
\hline$p_{p}, q, r$ & $\begin{aligned}= & \text { no-roll frame components of projectile angular } \\
& \text { velocity vector with respect to the inertial frame }\end{aligned}$ \\
\hline$p_{L}$ & $=$ spin rate of liquid \\
\hline$u, v, w$ & $\begin{array}{l}=\text { no-roll frame components of the velocity of the } \\
\text { body center of mass with respect to the inertial } \\
\text { frame }\end{array}$ \\
\hline$V$ & $\begin{aligned}= & \text { total velocity of the projectile center of mass with } \\
& \text { respect to the inertial frame }\end{aligned}$ \\
\hline$X, Y, Z$ & total external forces applied to the projectile \\
\hline$x, y, z$ & projectile position coordinates \\
\hline$\rho$ & atmospheric air density \\
\hline
\end{tabular}

Received 5 December 2011; revision received 15 March 2012; accepted for publication 27 March 2012. Copyright (C 2012 by the American Institute of Aeronautics and Astronautics, Inc. All rights reserved. Copies of this paper may be made for personal or internal use, on condition that the copier pay the $\$ 10.00$ per-copy fee to the Copyright Clearance Center, Inc., 222 Rosewood Drive, Danvers, MA 01923; include the code 0022-4650/13 and \$10.00 in correspondence with the CCC.

${ }^{*}$ Assistant Professor, Department of Aerospace Engineering. Member AIAA.

†Sikorsky Associate Professor, School of Aerospace Engineering. Associate Fellow AIAA.

${ }^{\ddagger}$ Physicist, Weapons and Materials Research Directorate. Senior Member AIAA.

$$
\begin{aligned}
\tau_{F}, \tau_{S}= & \text { projectile fast and slow epicyclic frequencies } \\
& \text { nondimensionalized by } p_{L} \\
\phi_{P}, \theta, \psi= & \text { projectile Euler roll, pitch, and yaw rotation } \\
& \text { angles } \\
\phi_{L} & \text { liquid Euler roll angle } \\
\Omega_{F}, \Omega_{S}= & \text { dimensional projectile fast and slow epicyclic } \\
& \text { frequencies }
\end{aligned}
$$

\section{Introduction}

$\mathbf{P}^{\mathrm{n}}$ ROJECTILES containing liquid payloads are currently employed in a variety of applications. Shells filled with white phosphorus are commonly used in battlefield scenarios to generate smoke screens [1_-4], while other 'less-than-lethal' projectiles may deliver a liquid payload to a target [5]. New hollow-shell designs may carry medical liquids such as intravenous fluid bags for humanitarian missions [6]. Historically, projectiles containing liquid payloads have exhibited significantly different flight behavior in comparison to solid projectiles. The motion of the liquid within the body can generate large moments on the projectile, which can prematurely terminate the flight by causing instability. Repeated observation of this phenomenon has demonstrated that sharp increases in angle of attack (AOA) and large reductions in spin rate are symptomatic of liquid-induced instability [ㄱ-10].

Numerous authors have attempted to predict the onset of fluid instabilities in a gun-launched projectile, primarily through the use of computational fluid dynamic (CFD) theories and linearized fluid theories based on spatial eigenvalue methods. One limitation of CFD solutions is that simulation of fluids at high Reynolds numbers $(R e)$ can be problematic due to spatial resolution requirements, and thus many investigators have turned to analytical spatial-eigenvalue methods that can capture a broader range of $R e$. Sedney [11] provides a comprehensive survey of liquid payload projectile flight dynamics and the nature of liquid-induced instabilities. Several authors have made use of analytic techniques that use inviscid fluid theory with viscous boundary-layer corrections [12-14]. Weber [15] made use of tricyclic projectile linear theory, assuming the effect of liquid moments was similar to the Magnus effect. Vaughn et al. [16] simulated projectile angular motion for fluids at low Re by integrating CFD results into 6-degree-of-freedom (DOF) calculations. Recently, Cooper and Costello [17] used a well-developed spatial eigenvalue theory to generate liquid moment coefficients and integrated them 
into a full 6DOF flight model. The analysis assumed that the projectile undergoes quasi-steady coning motion, which enables calculation of moment coefficients as a function of $R e$ and coning frequency from the linearized Navier-Stokes equations.

Extensive work has also been performed on the related problem of instabilities observed in spacecraft equipped with liquid-filled fuel tanks. An interesting and comprehensive survey of spacecraft-related rotating liquids work is provided by Abramson [18]. As in the projectile community, difficulties with dynamic modeling of rotating liquid payloads led to the use of experimental fixtures aimed at understanding and predicting liquid resonances in fuel tanks onboard spinning spacecraft. Pocha [19] found that resonances in rotationally symmetric tanks were highly damped with the introduction of circular baffle plates installed orthogonal to the symmetry axis. Agrawal [20] developed a numerical model predicting liquid motion in partially filled tanks, combining inviscid fluid theory with boundary-layer and viscous corrections. Manasseh [21] experimentally confirmed the effectiveness of internal tank baffles but only when they were designed with optimal size and configuration. Deffenbaugh et al. [22] describe an experimental investigation of rotating liquids in spacecraft fuel tanks on STS 84 in 1997, providing further empirical data that characterized rotating liquid resonances in a space environment. Kobine [23] attempted to experimentally characterize the accuracy of linear and inviscid approximations typically used in rotating liquid models, demonstrating that such approximations were generally valid. However, it was noted that boundary-layer phenomena become increasingly important as the fluid approaches resonance. Bao and Pascal [24] and Gerrits and Veldman [25] have developed additional models and stability analysis for sloshing in partially filled spacecraft tanks. It is important to note that a primary feature distinguishing rotating liquids research related to projectiles and spacecraft is that typical spacecraft spin rates are 1-2 orders of magnitude less than standard spin-stabilized artillery rounds, which can lead to significant differences to the range of $R e$ encountered in the problem.

This paper extends the work of Cooper and Costello [17] by increasing model fidelity and studying the effects of various design parameters on liquid-induced instability for spin-stabilized projectiles. Model fidelity is enhanced first by using a dual-spin projectile model to capture liquid spin-up after launch. Second, the model separates fast and slow coning motion within the flight simulation, enabling more-accurate calculation of liquid moments due to fast and slow epicyclic motion. Through the use of projectile linear theory, fast- and slow-mode angles of attack are determined at each time step using a batch linear least-squares filter operating simultaneously with the dynamic simulation. The paper begins with a description of the liquid payload projectile model and the angle-ofattack filter. Example results show how fast-mode angle of attack created by launch disturbances leads to liquid instability, even when tipoff perturbations are relatively small. Then, Monte Carlo simulations demonstrate the effects of liquid cavity geometry, liquid spin-up rate, and magnitude of launch perturbations on the likelihood of instability occurring. Analysis of these results leads to the conclusion that lower cavity aspect ratios and higher launch perturbations leads to a higher chance of instability, while slower liquid spin-up rates lead to less observed instability.

\section{Projectile Dynamic Model}

The projectile model used here is a 7DOF flight dynamic model. The liquid payload within the projectile is considered to have a uniform spin rate and is allowed to spin independently from the projectile. Dynamic interaction between the liquid and the projectile is governed by liquid moments, the computation of which is described in detail in the following section. These liquid moments combine with aerodynamic and gravity effects to drive projectile motion.

Three reference frames are used in the dynamic modeling process, namely the inertial reference frame, the body reference frame, and the no-roll reference frame. The inertial reference frame is aligned such that $\mathbf{I}_{I}$ lies along the gun line of fire and makes use of the flat earth

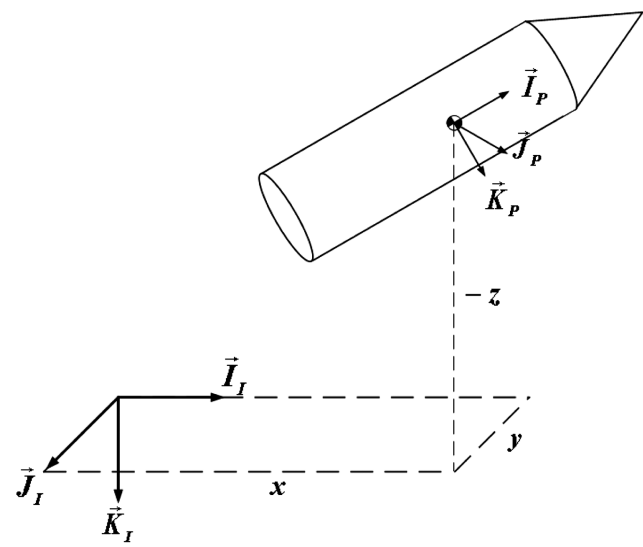

Fig. 1 Projectile position coordinate definitions.

approximation. The body reference frame is obtained through the standard Euler angle rotation sequence, where $\phi_{p}, \theta$, and $\psi$ denote projectile roll, pitch, and yaw angles, respectively. The no-roll frame is obtained by rotating the body frame by $-\phi_{p}$ about $\mathbf{I}_{P}$. Position and coordinate definitions are shown in Fig. 1 .

\section{A. Equations of Motion}

The dynamic model used here differs from standard 6DOF dynamic models in that the projectile is assumed to be composed of two bodies coupled by a bearing containing viscous friction. These separate outer and inner bodies can rotate with respect to one another about the $\mathbf{I}_{P}$ axis. The outer body represents the projectile while the inner body represents the liquid spinning at a uniform rate. Thus, the simulation can model uniform, time-dependent fluid spin-up, and the viscous bearing friction can be adjusted so as to generate a realistic spin-up profile. The 7DOF equations of motion are presented in Eqs. (1- $\underline{4})$ :

$$
\begin{gathered}
\left\{\begin{array}{c}
\dot{x} \\
\dot{y} \\
\dot{z}
\end{array}\right\}=\left[\begin{array}{ccc}
c_{\theta} c_{\psi} & -s_{\psi} & s_{\theta} c_{\psi} \\
c_{\theta} s_{\psi} & c_{\psi} & s_{\theta} s_{\psi} \\
-s_{\theta} & 0 & c_{\theta}
\end{array}\right]\left\{\begin{array}{c}
u \\
v \\
w
\end{array}\right\} \\
\left\{\begin{array}{c}
\dot{\phi}_{P} \\
\dot{\phi}_{L} \\
\dot{\theta} \\
\dot{\psi}
\end{array}\right\}=\left[\begin{array}{cccc}
1 & 0 & 0 & t_{\theta} \\
0 & 1 & 0 & t_{\theta} \\
0 & 0 & 1 & 0 \\
0 & 0 & 0 & 1 / c_{\theta}
\end{array}\right]\left\{\begin{array}{c}
p_{P} \\
p_{L} \\
q \\
r
\end{array}\right\} \\
\left\{\begin{array}{c}
\dot{u} \\
\dot{v} \\
\dot{w}
\end{array}\right\}=\left\{\begin{array}{c}
X / m \\
Y / m \\
Z / m
\end{array}\right\}-\left[\begin{array}{ccc}
0 & -r \\
r & 0 \\
-q & r t_{\theta} & 0
\end{array}\right]\left\{\begin{array}{c}
u \\
v \\
w
\end{array}\right\} \\
\left\{\begin{array}{c}
t_{\theta} \\
\dot{p}_{P} \\
\dot{p}_{L} \\
\dot{q} \\
\dot{r}
\end{array}\right\}=[I]^{-1}\left\{\begin{array}{c}
g_{P 1}-M_{V} \\
g_{L 1}+M_{V} \\
M_{2}-S_{2}^{*} \\
M_{3}-S_{3}^{*}
\end{array}\right\}
\end{gathered}
$$

Note that, in Eqs. (1- $\underline{4})$, the quantities $\phi_{p}$ and $\phi_{L}$ denote projectile and liquid roll angles, respectively, and $p_{P}$ and $p_{L}$ denote projectile and liquid spin rates, respectively. Also, the following shorthand notation for trigonometric functions is used: $s_{\alpha}=\sin (\alpha)$, $c_{\alpha}=\cos (\alpha)$, and $t_{\alpha}=\tan (\alpha)$. In Eq. (4), the right-hand-side vector contains lengthy expressions that are developed in detail in [26]. Derivation of Eq. (4) is accomplished by solving moment balance equations for the projectile about all three body axes and a single moment balance equation on the liquid payload about $\mathbf{I}_{P}$. A detailed derivation of the dual-spin equations is somewhat lengthy and beyond the scope of this paper but is shown in [26]. Given a set of initial conditions, Eqs. (1-4) are integrated forward in time using a fourth-order Runge--Kutta algorithm to obtain a single trajectory. 


\section{B. Forces and Moments}

Projectile motion is driven by aerodynamics, gravity, and the interaction between the liquid payload and the projectile body. However, the liquid payload is assumed to exert only a moment on the body, and thus the total forces on the projectile $(X, Y, Z)$ can be represented by

$$
\left\{\begin{array}{c}
X \\
Y \\
Z
\end{array}\right\}=\left\{\begin{array}{c}
X_{A} \\
Y_{A} \\
Z_{A}
\end{array}\right\}+\left\{\begin{array}{c}
X_{M} \\
Y_{M} \\
Z_{M}
\end{array}\right\}+\left\{\begin{array}{c}
X_{W} \\
Y_{W} \\
Z_{W}
\end{array}\right\}
$$

where $A, M$, and $W$ subscripts represent aerodynamic, Magnus, and weight forces, respectively. Components of the weight force are given by

$$
\left\{\begin{array}{c}
X_{W} \\
Y_{W} \\
Z_{W}
\end{array}\right\}=\operatorname{mg}\left\{\begin{array}{c}
-s_{\theta} \\
s_{\phi_{P}} c_{\theta} \\
c_{\phi_{P}} c_{\theta}
\end{array}\right\}
$$

Aerodynamic and Magnus forces are a function of angle of attack and local Mach number and are based on the Projectile Rocket Ordnance Design and Analysis System of aerodynamic expansion. A detailed description of aerodynamic forces is provided in [17] and is omitted here for brevity.

The total moments exerted on the projectile $(L, M, N)$ are comprised of steady aerodynamic $(S)$, unsteady aerodynamic $(U)$, Magnus $(M)$, and liquid terms $(L)$, and are given by

$$
\left\{\begin{array}{c}
L \\
M \\
N
\end{array}\right\}=\left\{\begin{array}{c}
L_{S} \\
M_{S} \\
N_{S}
\end{array}\right\}+\left\{\begin{array}{c}
L_{U} \\
M_{U} \\
N_{U}
\end{array}\right\}+\left\{\begin{array}{c}
L_{M} \\
M_{M} \\
N_{M}
\end{array}\right\}+\left\{\begin{array}{c}
L_{L} \\
M_{L} \\
N_{L}
\end{array}\right\}
$$

Liquid moment terms will be discussed in the next section. Steady aerodynamic moments are obtained be computing the cross between the distance vector from the center of gravity to the center of pressure and the body aerodynamic force vector. Likewise, the Magnus aerodynamic moment is computed with a cross product between the distance vector from the center of mass to the center of Magnus force and the Magnus force vector. The unsteady body aerodynamic moment provides a damping source for projectile angular motion. Detailed expressions for these damping moments are omitted here for brevity but are provided in [17]. All aerodynamic coefficients and aerodynamic center distances are a function of local Mach number at the center of mass of the projectile. Computationally, these Mach number-dependent parameters are obtained during vehicle simulation by a table lookup scheme using linear interpolation.

\section{Liquid Payload Model}

Angular motion of the projectile is altered by moments generated from inertial waves within the liquid payload. These waves impact the walls of the liquid cavity and produce so-called liquid moments on the projectile body. Murphy et al. [27-29] showed that, in some cases, these liquid moments can have a devastating impact on the projectile's angular motion. This section describes the computation of liquid moments within the flight-simulation model.

\section{A. Liquid Moment Computation}

There are several underlying assumptions behind computation of the liquid moments at each time step. The first is that the projectile is continually undergoing steady-state coning motion with a given magnitude and frequency. Thus, although the liquid spin rate is considered to be time-dependent and is driven by the dual-spin model presented previously, other transient motion of the liquid is neglected, and it is assumed to be constantly in steady state. This assumption is critical in generating liquid moment coefficients without having to resort to CFD, which is difficult to implement for the high Reynolds numbers required here. The second assumption is that the liquid cavity can be represented by a cylinder with an equivalent aspect ratio. This assumption provides closed-form solutions for the linearized Navier-Stokes equations that enable generation of liquid moment coefficients.

The quasi-steady assumption described previously requires that the liquid payload undergo identical angular motion to that of the projectile (with the exception of its spin rate, which is treated separately through the use of the dual-spin model). According to wellestablished linear theory [27], angular motion can be decomposed into fast and slow epicyclic modes. These modes generate inertial wave motion within the liquid that interacts with the projectile body. Thus, the liquid moment can be represented as the sum of moments caused by fast-mode coning and those caused by slow-mode coning, as shown in Eq. (

$$
\left\{\begin{array}{c}
L_{L} \\
M_{L} \\
N_{L}
\end{array}\right\}=\left\{\begin{array}{c}
L_{L F} \\
M_{L F} \\
N_{L F}
\end{array}\right\}+\left\{\begin{array}{c}
L_{L S} \\
M_{L S} \\
N_{L S}
\end{array}\right\}
$$

Fast and slow liquid moments are computed according to

$$
\begin{gathered}
\left\{\begin{array}{c}
L_{L F} \\
M_{L F} \\
N_{L F}
\end{array}\right\}=m_{L} a^{2} p_{L}^{2} \tau_{F}\left[\begin{array}{ccc}
1 & 0 & 0 \\
0 & c_{\beta} & s_{\beta} \\
0 & -s_{\beta} & c_{\beta}
\end{array}\right]\left\{\begin{array}{c}
\alpha_{F}^{2} C_{\mathrm{LRM}_{F}} \\
\alpha_{F} C_{\mathrm{LSM}_{F}} \\
\alpha_{F} C_{\mathrm{LIM}_{F}}
\end{array}\right\} \\
\left\{\begin{array}{c}
L_{L S} \\
M_{L S} \\
N_{L S}
\end{array}\right\}=m_{L} a^{2} p_{L}^{2} \tau_{S}\left[\begin{array}{ccc}
1 & 0 & 0 \\
0 & c_{\beta} & s_{\beta} \\
0 & -s_{\beta} & c_{\beta}
\end{array}\right]\left\{\begin{array}{c}
\alpha_{S}^{2} C_{\mathrm{LRM}_{S}} \\
\alpha_{S} C_{\mathrm{LSM}_{S}} \\
\alpha_{S} C_{\mathrm{LIM}_{S}}
\end{array}\right\}
\end{gathered}
$$

where $\beta=v / w, m_{L}$ represents the mass of the liquid, $a$ is the equivalent cylinder radius, and $\tau_{S}$ and $\tau_{F}$ are the slow and fast epicyclic frequencies nondimensionalized by the liquid spin rate $p_{L}$. Coefficients $C_{\mathrm{LRM}}, C_{\mathrm{LSM}}$, and $C_{\mathrm{LIM}}$ are liquid moment coefficients and will be discussed in detail in a following section. Projectile epicyclic frequencies are calculated at each time step using the expressions given in [30]. Note that Eqs. (9) and (10) arise from a substantial literature base that incorporates both analytical models as well as experimental results [27-29].

\section{B. Fast- and Slow-Mode Angles of Attack}

Equations (9) and (10) require determination of the fast- and slowmode angles of attack at each computation cycle. In general, projectile coning motion is a combination of a trim angle as well as fast-mode and slow-mode coning. The polar plot shown in Fig. 2, generated from a flight simulation of a representative spin-stabilized projectile, provides a visual representation of how projectile coning motion occurs. The figure shows the path of the projectile nose with respect to the velocity vector. Note that slow-mode coning is an elliptical motion of the nose about a trim angle of attack, whereas

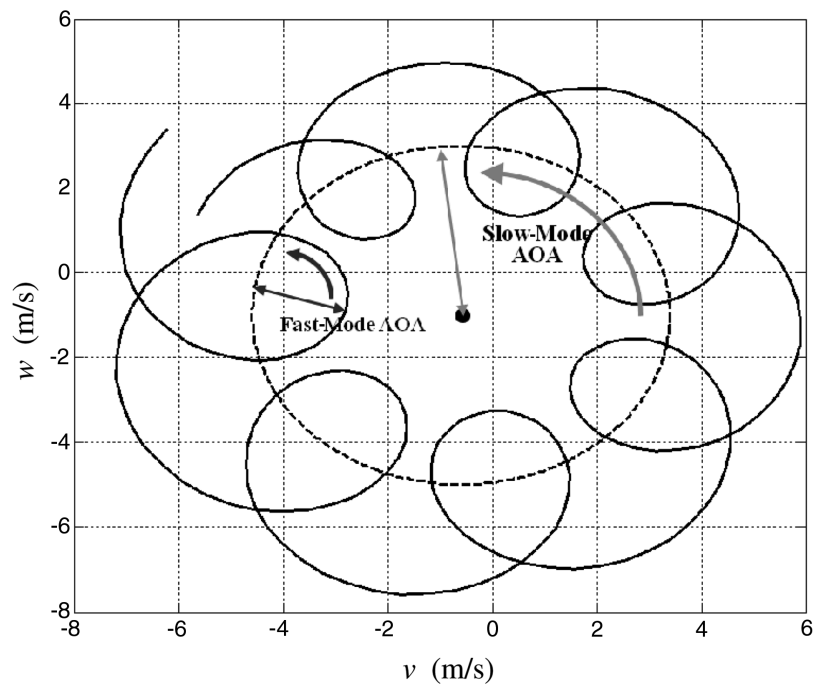

Fig. 2 Polar plot showing fast- and slow-mode coning about a trim angle for representative spin-stabilized projectile. 
fast-mode coning occurs about an order of magnitude faster and involves elliptical motion around the slow-mode coning profile. The frequencies of fast and slow coning motion are provided by projectile linear theory [30]. However, the magnitude of each of these coning motions must be determined at each time step to calculate $\alpha_{F}$ and $\alpha_{S}$.

To calculate $\alpha_{F}$ and $\alpha_{S}$, the no-roll frame velocity components $v$ and $w$ must be separated into fast- and slow-mode components. This is accomplished using a batch linear least-squares filter applied at discrete intervals. The linear theory solution for velocity components $v$ and $w$ is given by

$$
\begin{aligned}
v & =v_{F S} \sin \left(\Omega_{F} t\right)+v_{F C} \sin \left(\Omega_{F} t\right)+v_{S S} \sin \left(\Omega_{S} t\right) \\
& +v_{S C} \sin \left(\Omega_{S} t\right)+v_{T} \\
w & =w_{F S} \sin \left(\Omega_{F} t\right)+w_{F C} \sin \left(\Omega_{F} t\right)+w_{S S} \sin \left(\Omega_{S} t\right) \\
& +w_{S C} \sin \left(\Omega_{S} t\right)+w_{T}
\end{aligned}
$$

where $\Omega_{F}$ and $\Omega_{S}$ are the dimensional fast and slow epicyclic frequencies, respectively. Given a window of $N$ previous values for $v$ and $w$, the following linear systems can be created at each time step, assuming $\Omega_{F}$ and $\Omega_{S}$ are constant throughout the time history:

$$
\begin{aligned}
& \left\{\begin{array}{c}
v_{1} \\
\vdots \\
v_{N}
\end{array}\right\} \\
& =\left[\begin{array}{ccccc}
\sin \left(\Omega_{F} t_{1}\right) & \cos \left(\Omega_{F} t_{1}\right) & \sin \left(\Omega_{S} t_{1}\right) & \cos \left(\Omega_{S} t_{1}\right) & 1 \\
\vdots & \vdots & \vdots & \vdots & \vdots \\
\sin \left(\Omega_{F} t_{N}\right) & \cos \left(\Omega_{F} t_{N}\right) & \sin \left(\Omega_{S} t_{N}\right) & \cos \left(\Omega_{S} t_{N}\right) & 1
\end{array}\right] \\
& \times\left\{\begin{array}{c}
v_{F S} \\
v_{F C} \\
v_{S S} \\
v_{S C} \\
v_{T}
\end{array}\right\}=[H]\left\{\begin{array}{c}
v_{F S} \\
v_{F C} \\
v_{S S} \\
v_{S C} \\
v_{T}
\end{array}\right\}
\end{aligned}
$$

$$
\begin{aligned}
& \left\{\begin{array}{c}
w_{1} \\
\vdots \\
w_{N}
\end{array}\right\} \\
& =\left[\begin{array}{ccccc}
\sin \left(\Omega_{F} t_{1}\right) & \cos \left(\Omega_{F} t_{1}\right) & \sin \left(\Omega_{S} t_{1}\right) & \cos \left(\Omega_{S} t_{1}\right) & 1 \\
\vdots & \vdots & \vdots & \vdots & \vdots \\
\sin \left(\Omega_{F} t_{N}\right) & \cos \left(\Omega_{F} t_{N}\right) & \sin \left(\Omega_{S} t_{N}\right) & \cos \left(\Omega_{S} t_{N}\right) & 1
\end{array}\right] \\
& \times\left\{\begin{array}{c}
w_{F S} \\
w_{F C} \\
w_{S S} \\
w_{S C} \\
w_{T}
\end{array}\right\}=[H]\left\{\begin{array}{c}
w_{F S} \\
w_{F C} \\
w_{S S} \\
w_{S C} \\
w_{T}
\end{array}\right\}
\end{aligned}
$$

where subscripts $1, \ldots N$ denote values in the time history. The optimal least-squares solution to each of these linear systems is given by

$$
\left\{\begin{array}{c}
v_{F S} \\
v_{F C} \\
v_{S S} \\
v_{S C} \\
v_{T}
\end{array}\right\}=\left(H^{T} H\right)^{-1} H^{T}\left\{\begin{array}{c}
v_{1} \\
\vdots \\
v_{N}
\end{array}\right\}
$$

$$
\left\{\begin{array}{c}
w_{F S} \\
w_{F C} \\
w_{S S} \\
w_{S C} \\
w_{T}
\end{array}\right\}=\left(H^{T} H\right)^{-1} H^{T}\left\{\begin{array}{c}
w_{1} \\
\vdots \\
w_{N}
\end{array}\right\}
$$

Note that some error is, of course, introduced by the assumption that $\Omega_{F}$ and $\Omega_{S}$ are constant throughout the filtering window, although in practice these frequencies change slowly compared to the filtering rate. Equations (15) and (16) are used at each time step to solve for the 10 parameters on the left-hand sides of both equations. Then, fastand slow-mode angles of attack are computed according to

$$
\begin{gathered}
\alpha_{F}=\frac{\sqrt{v_{F S}^{2}+v_{F C}^{2}}+\sqrt{w_{F S}^{2}+w_{F C}^{2}}}{2 u} \\
\alpha_{S}=\frac{\sqrt{v_{S S}^{2}+v_{S C}^{2}}+\sqrt{w_{S S}^{2}+w_{S C}^{2}}}{2 u}
\end{gathered}
$$

These angles of attack are then used in Eqs. (9) and (10) to compute the liquid moments.

\section{Liquid Resonances}

Liquid moment coefficients $C_{\mathrm{LSM}}$ and $C_{\mathrm{LIM}}$ used in Eqs. (9) and (10) are obtained by solving the linearized Navier-Stokes equations for a projectile in steady-state coning motion. Viscous boundarylayer corrections are applied near the cavity walls, yielding a closedform inertial wave solution for the cylindrical payload container. These inertial wave solutions are then processed to obtain the time derivative of the liquid payload angular momentum, which yields the coefficients $C_{\mathrm{LSM}}$ and $C_{\mathrm{LIM}}$ for a given coning motion, liquid $R e$, and cylinder aspect ratio. At each time step, the current liquid $R e$, fastmode frequency, and slow-mode frequency are used to calculate $C_{\mathrm{LSM}}$ and $C_{\mathrm{LIM}}$ through linear interpolation. Note that the roll moment coefficient is obtained simply as $C_{\mathrm{LRM}}=-C_{\mathrm{LSM}}$ [28].

The phenomenon that leads to liquid instability is a resonance that occurs between the projectile coning motion and the inertial waves that propagate through the liquid. The frequency at which these resonance peaks occur is highly sensitive to cavity aspect ratio, while the magnitude depends on both aspect ratio and $\sqrt{R e}$. Figure 3 shows side moment coefficient $C_{\mathrm{LSM}}$, the coefficient most responsible for causing liquid instability, as a function of nondimensional coning frequency $\tau$ and $R e$. Note that the frequency at which the main resonance peak occurs shifts noticeably with aspect ratio. Figures 4 and 5 show $C_{\text {LIM }}$ for aspect ratios 1.15 and 4.2, respectively, again demonstrating a significant shift in resonance with aspect ratio.

The strong resonance peak in $C_{\mathrm{LSM}}$ shown in Fig. $\underline{3}$ is largely responsible for causing fast-mode liquid instability as observed repeatedly in liquid payload projectile flight tests. For spin-stabilized rounds, the nondimensional fast frequency given by

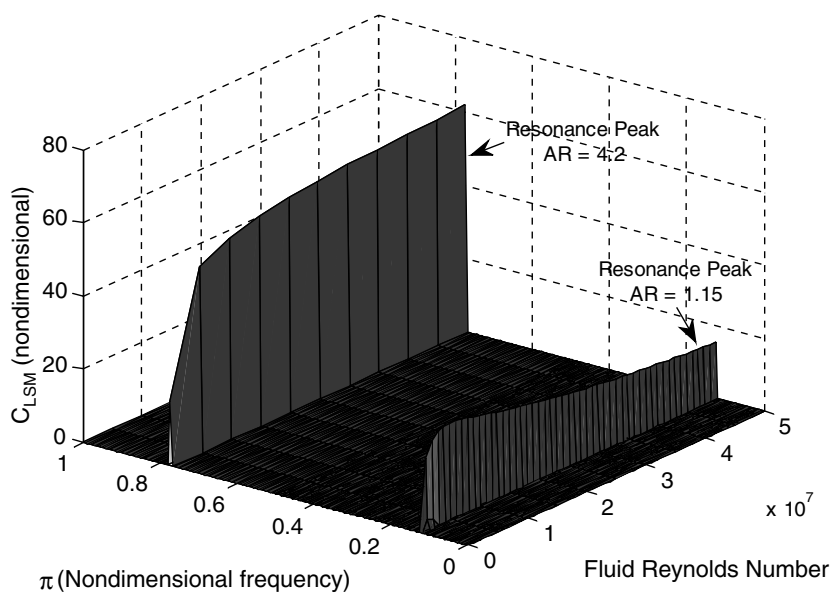

Fig. $3 C_{\mathrm{LSM}}$ vs $\tau$ and $R e$ for various cavity aspect ratios. 


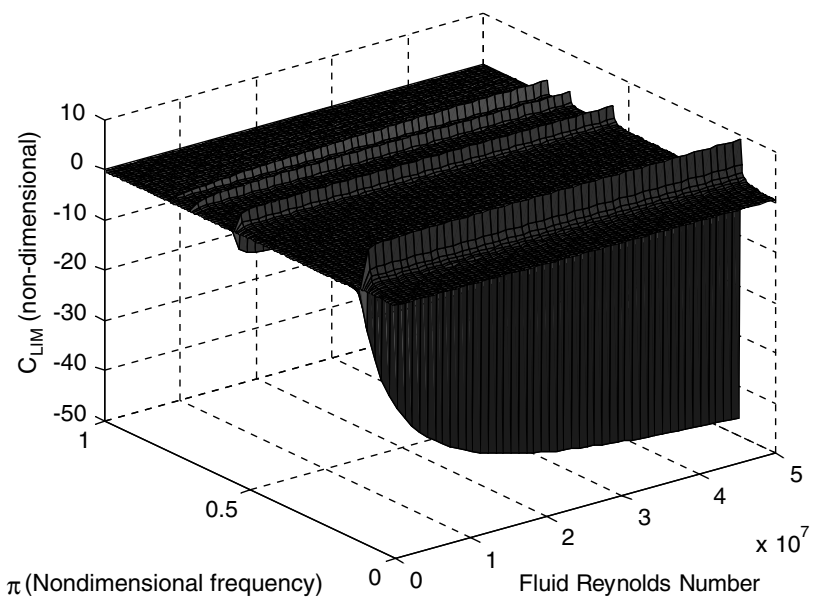

Fig. $4 C_{\mathrm{LIM}}$ vs $\tau$ and $R e$ for cavity aspect ratio 1.15 .

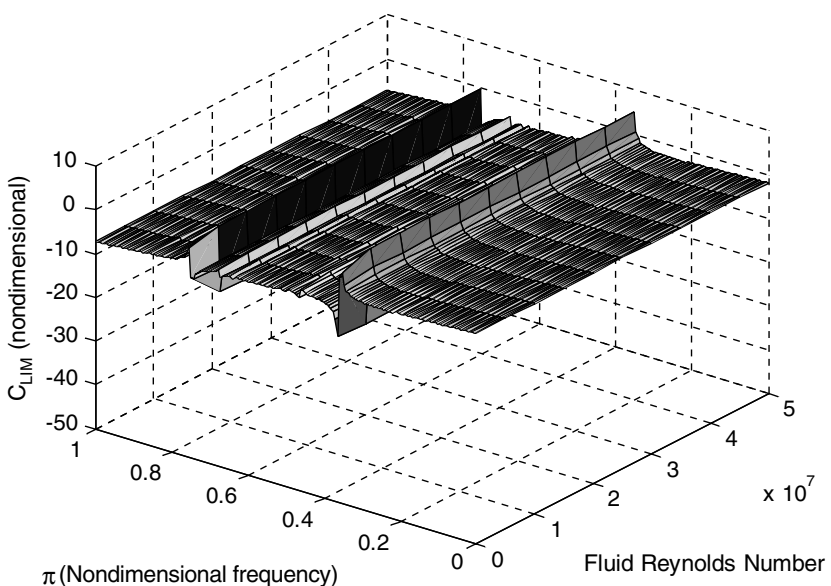

Fig. $5 C_{\mathrm{LIM}}$ vs $\tau$ and $R e$ for cavity aspect ratio 4.2.

$$
\tau_{F}=\frac{\Omega_{F}}{p_{L}}
$$

decreases quickly as the liquid spins up from values greater than 100 toward steady state values less than approximately 0.1 , passing through the resonance peak in the process. The spin-up time of the liquid, which varies with $\sqrt{R e}$, and the coning frequency at which these peaks occur are the two variables that determine the time after launch that the projectile passes through the resonance peak.

\section{Results}

This section describes example trajectories and Monte Carlo simulations used to evaluate model fidelity and the impact of specific design parameters of interest. The projectile used here is an indirect fire, spin-stabilized round with mass, axial inertia, and transverse inertia given by $43.00 \mathrm{~kg}, 0.14766 \mathrm{~kg} \cdot \mathrm{m}^{2}$, and $1.8990 \mathrm{~kg} \cdot \mathrm{m}^{2}$, respectively. For all trajectories in this section, nominal muzzle velocity is $826.0 \mathrm{~m} / \mathrm{s}$, quadrant elevation is $228.1 \mathrm{mils}$, and initial projectile spin rate is $1674 \mathrm{rad} / \mathrm{s}$. The liquid is assumed to have zero initial spin rate. A drawing of the example projectile is provided in Fig. 6. Note that, in all trajectories presented next, the projectile is assumed to be in unstable flight if total angle of attack exceeds $20 \mathrm{deg}$, and flight simulation is terminated at this time.

\section{A. Example Trajectories}

Two example simulations will be used to demonstrate the effects of the liquid payload on projectile flight. The first example, in which instability does not occur, contrasts the flight of the example projectile with liquid and solid payloads. The second example shows

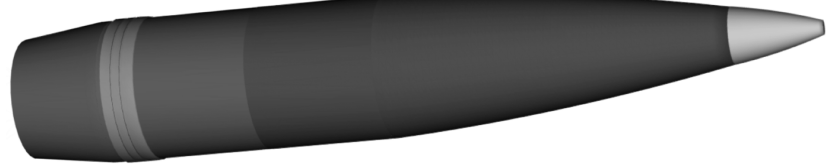

Fig. 6 Example spin-stabilized projectile.

a liquid payload flight in which instability does occur. Initial conditions for both examples include perturbed initial angles of attack and angular rates, as outlined in Table 1 . All example trajectories use a cavity aspect ratio of 1.118 .

Figures 7 and 8 show altitude-range and deflection-range plots for both the liquid and solid payload trajectories. Note that the trajectories are overlaid in each figure. Figure 9 shows a time history of total angle of attack for the liquid and solid cases. In the liquid case, the fast-mode liquid resonance is encountered at approximately $5 \mathrm{~s}$, leading to a temporary slight increase in fast-mode angle of attack oscillations. Figure 10 shows the slow- and fast-mode angles of attack output from the linear filtering algorithm for the liquid payload case. Again, note the spike in fast-mode angle of attack around $5 \mathrm{~s}$ caused by liquid resonance. Figure 11 , a spin-rate time history for the liquid payload case, shows that the liquid achieves steady-state spin approximately 15-20 s into flight. This spin-up time is assessed to be reasonable for the liquid and geometry considered here based on rough estimates generated from fundamental one-dimensional calculations. Figure 12 shows a time history of projectile epicyclic frequencies for the liquid payload case. Figure 13 shows a time history of the fast-mode nondimensional frequency $\left(\tau_{F}\right)$ for the liquid payload case. Notice that, for the aspect ratio of 1.118 used here, the liquid resonance occurs near $\tau_{F}=0.15$, which is encountered approximately $5 \mathrm{~s}$ into the trajectory. This is demonstrated by the liquid moment coefficient time history shown in Fig. 14, which shows that the projectile is excited by the fast-mode resonance for approximately $1 \mathrm{~s}$. The resulting liquid moments can be seen in Fig. 15 .

Although in the first example liquid moments did cause some changes in projectile dynamic behavior after encountering fluid resonance, these moments are not large enough to result in catastrophic instability. In the second example, slight changes are made to the initial launch perturbations, and only the liquid payload case is considered (the solid payload case proved to be stable with a flight profile similar to example 1 and thus is not shown here). These small increases in initial pitch and yaw rates induce a slightly higher fastmode angle of attack when liquid resonance is encountered. This creates larger liquid moments at resonance than in the first example. These moments generate an increase in fast-mode angle of attack, which in turn feed back to create higher overturning liquid moments. This positive feedback loop is what eventually causes instability in this second example. Figure 16 shows an altitude-range plot terminated at the location at which the projectile exceeds 20 deg total angle of attack. The fast- and slow-mode angles of attack shown in Figure 17 demonstrate that, although fluid resonance is encountered around $\overline{5 \mathrm{~s}}$, actual instability is not exhibited until after $9 \mathrm{~s}$. The period between 5 and $9 \mathrm{~s}$ is characterized by increasing fast-mode angle of attack and increasing liquid moments, which feed back on one another. Figure 18 shows a time history of the liquid moments. Note that, after liquid resonance is encountered, gradual growth in the noroll $Z$ component of the liquid moment occurs as the fast mode is excited, eventually leading to instability after $9 \mathrm{~s}$.

Table 1 Initial conditions for example trajectories

\begin{tabular}{lcc}
\hline \hline State & Example 1 & Example 2 \\
\hline$v, \mathrm{~m} / \mathrm{s}$ & -0.3 & -0.3 \\
$w, \mathrm{~m} / \mathrm{s}$ & 0.0 & 0.0 \\
$q, \mathrm{rad} / \mathrm{s}$ & 0.10 & 0.20 \\
$r, \mathrm{rad} / \mathrm{s}$ & -0.10 & -0.10 \\
\hline \hline
\end{tabular}




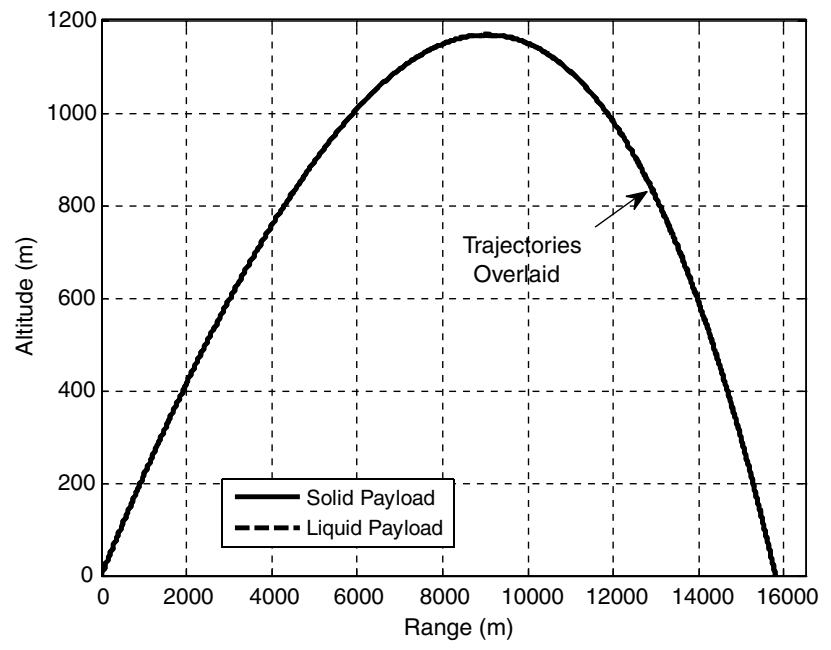

Fig. 7 Altitude vs range (stable example).

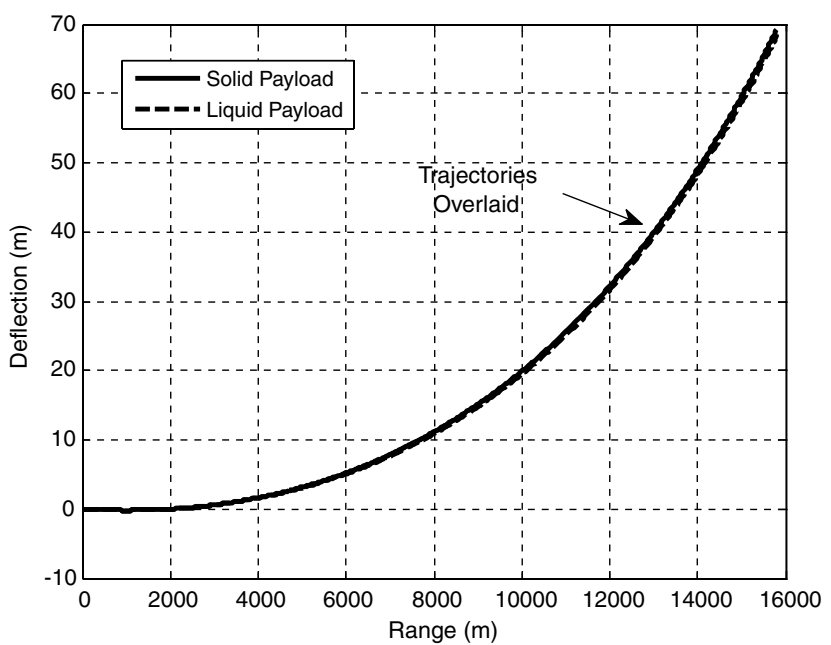

Fig. 8 Deflection vs range (stable example).

\section{B. Monte Carlo Simulations}

Three Monte Carlo simulations were performed to demonstrate the statistical nature of liquid-induced flight instability for varying levels of launch perturbations. For each Monte Carlo run, 500 trajectory simulations were performed using the nominal launch parameters outlined at the beginning of this section. Initial crossing velocities $v$ and $w$ as well as initial angular rates $q$ and $r$ were
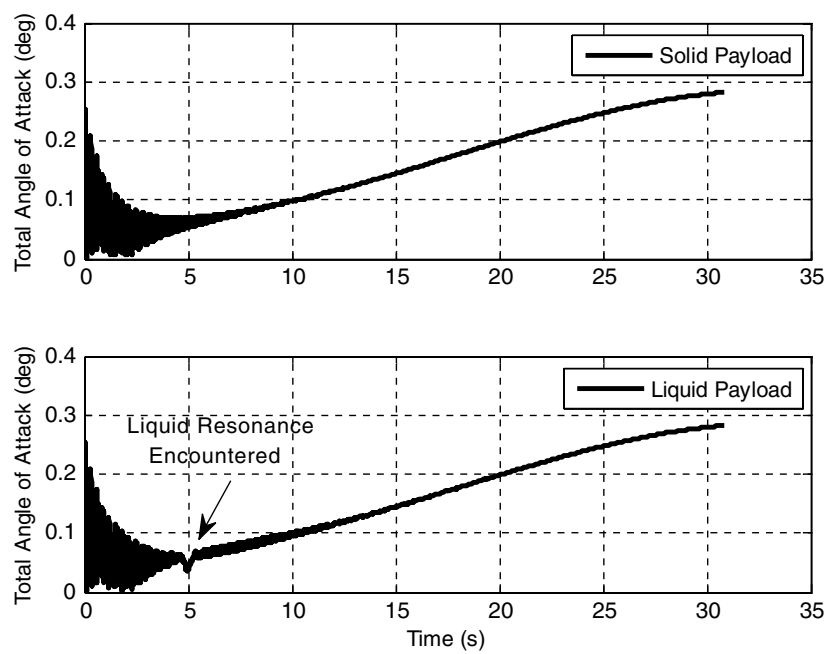

Fig. 9 Total angle of attack vs time (stable example).
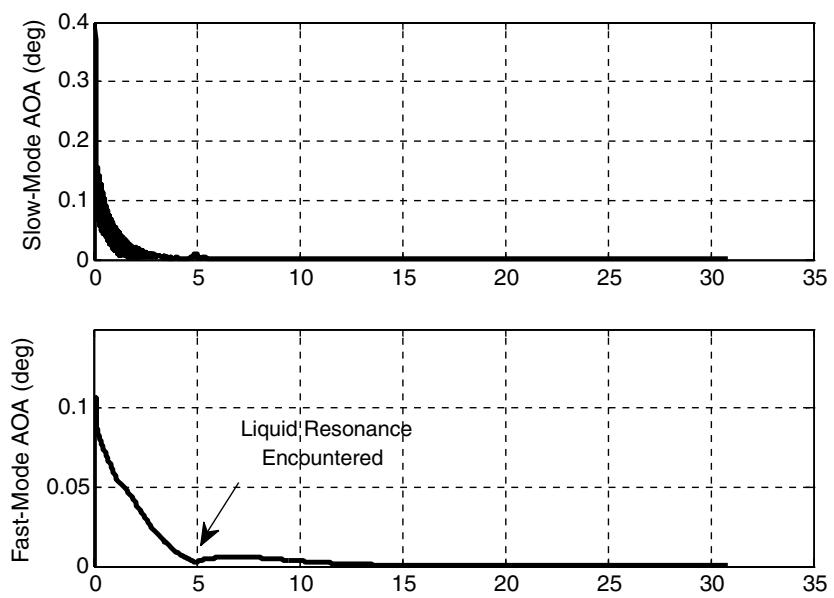

Fig. 10 Slow- and fast-mode angles of attack (liquid payload case, stable example).

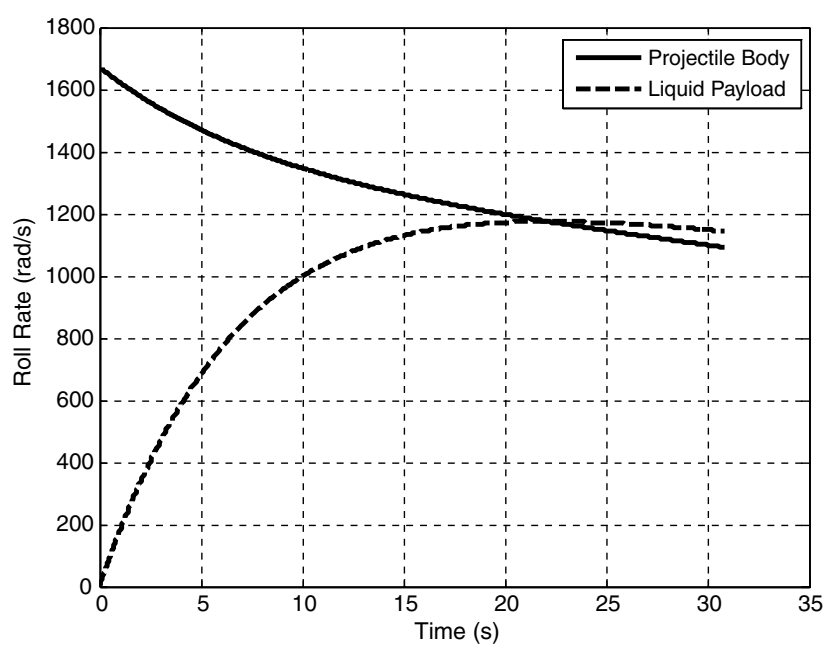

Fig. 11 Spin rate vs time for projectile body and liquid (liquid payload case, stable example).

perturbed according to zero-mean Gaussian distributions with standard deviations provided in Table 2 . In cases where instability occurred, the final state of the projectile was recorded, and the flight path was propagated to ground impact using a simple-vacuum-point mass model. The resulting impact locations provide a rough estimate of where such an unstable projectile would impact the ground. For

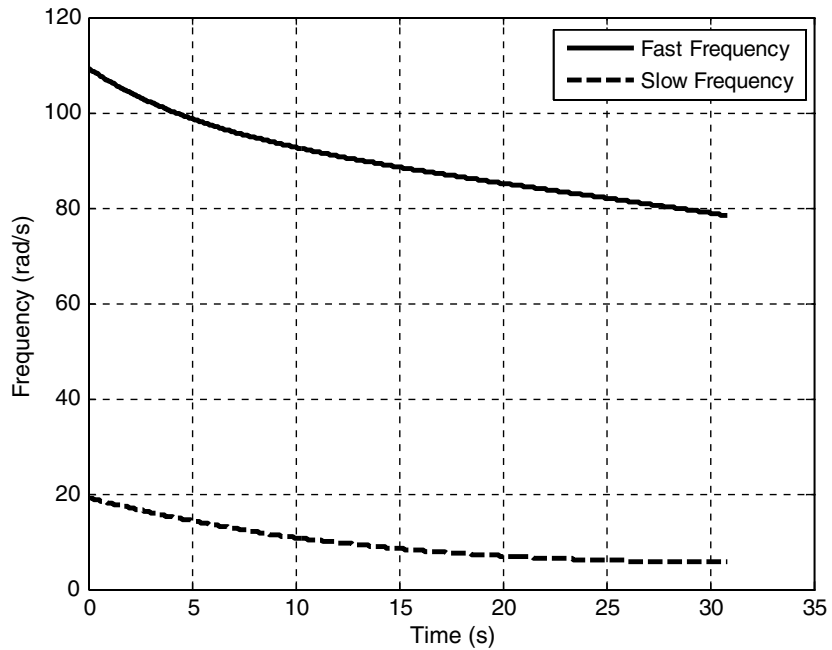

Fig. 12 Projectile epicyclic frequencies vs time (stable example). 


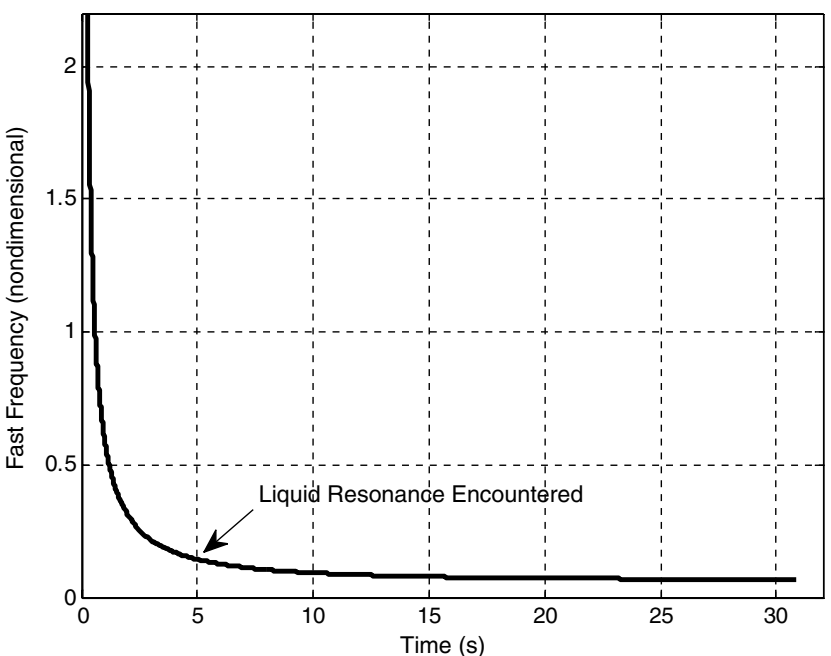

Fig. 13 Nondimensional fast frequency $\tau_{F}$ vs time (stable example).
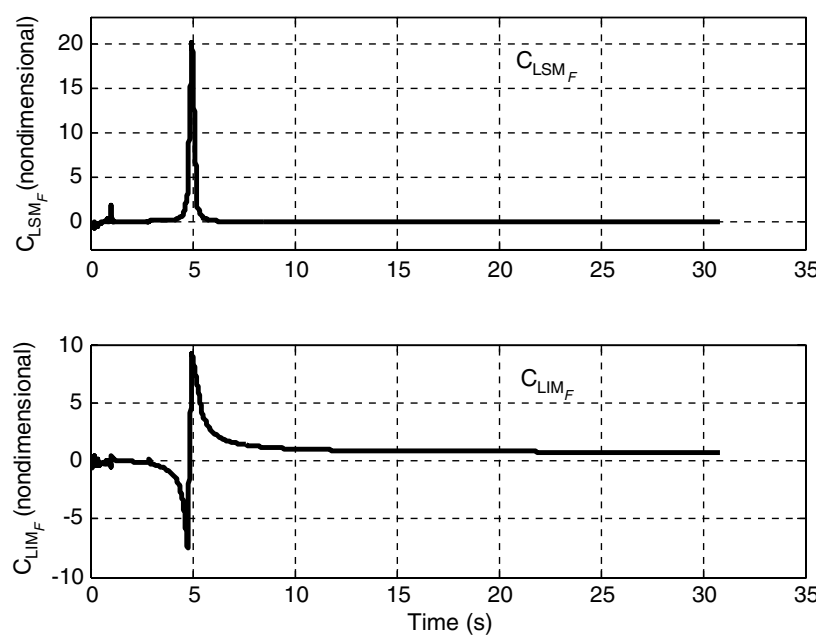

Fig. 14 Fast-mode liquid moment coefficients vs time (stable example).

each simulation, the number of cases exhibiting instability is shown in Table 2. Figure 19 shows impact point locations for all stable cases for Monte Carlo simulation 2. Stable impact dispersion patterns for simulations 1 and 3 were similar except for differing numbers of stable impacts and are not shown here. Note that a comparison of
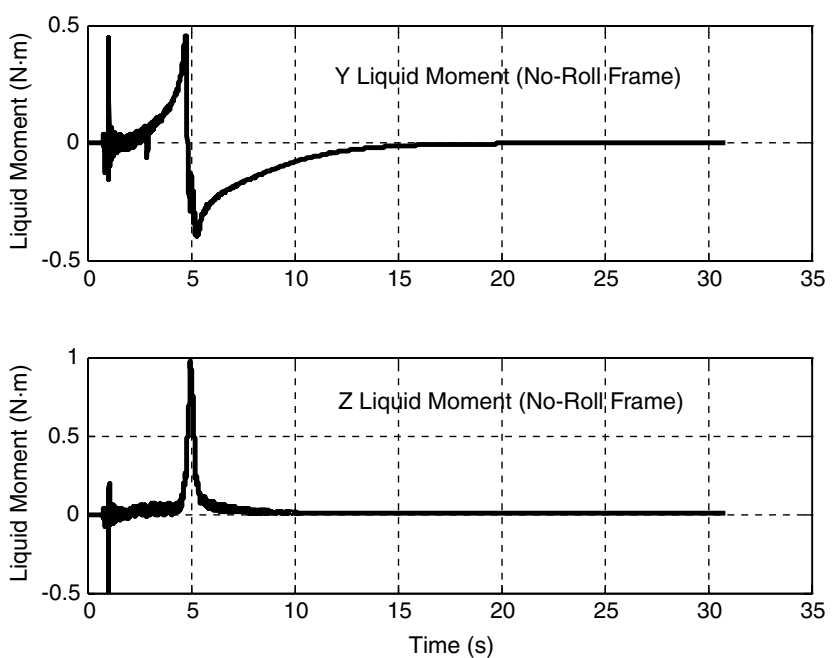

Fig. 15 Liquid moments vs time (stable example).

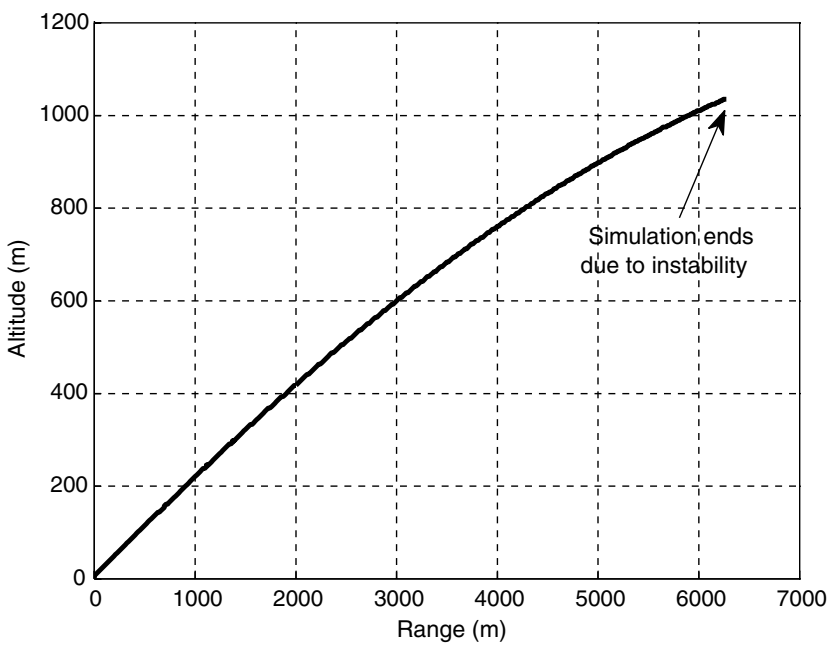

Fig. 16 Altitude vs range (unstable example).
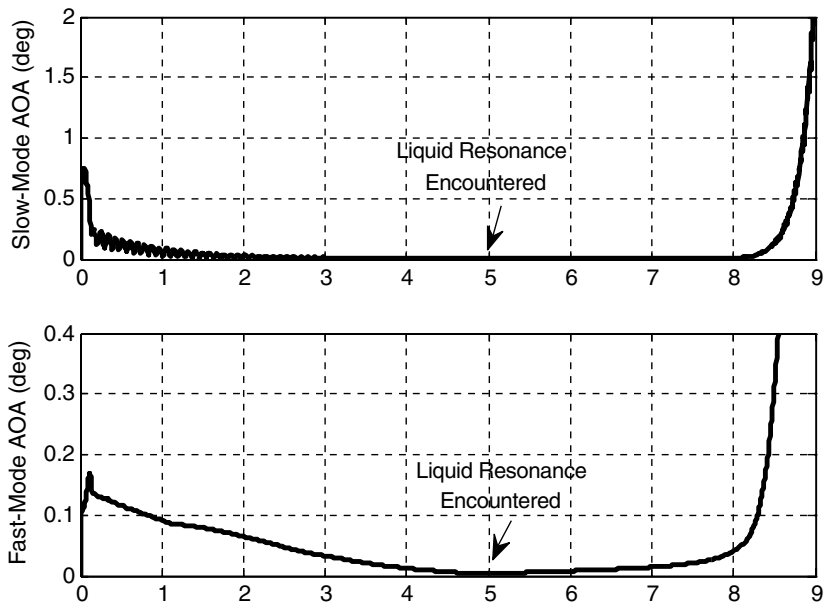

Fig. 17 Slow- and fast-mode angles of attack vs time (unstable example).

dispersion patterns between these stable liquid cases and those from a solid projectile showed almost no difference, signifying that liquid payload moments have little overall effect on the trajectory unless instability is induced. Figures 20-22 display the locations where instability was detected for the unstable cases as well as the
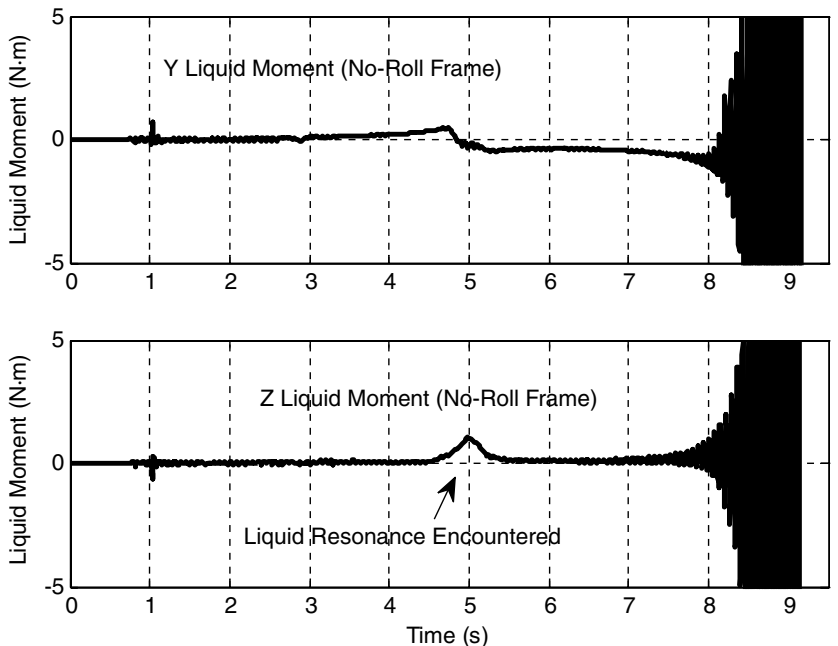

Fig. 18 Liquid moments vs time (unstable case). 
Table 2 Monte Carlo simulation parameters and results

\begin{tabular}{lccc}
\hline \hline $\begin{array}{c}\text { Monte Carlo } \\
\text { simulation } \\
\text { number }\end{array}$ & $\begin{array}{c}\text { Standard deviation } \\
\text { of initial velocity } \\
\text { errors }(v \text { and } w), \\
\mathrm{m} / \mathrm{s}\end{array}$ & $\begin{array}{c}\text { Standard deviation } \\
\text { of initial rate } \\
\text { errors }(q \text { and } r), \\
\text { rad } / \mathrm{s}\end{array}$ & $\begin{array}{c}\text { Number of } \\
\text { unstable } \\
\text { trajectories }\end{array}$ \\
\hline 1 & 0.15 & 0.10 & $186(37 \%)$ \\
2 & 0.20 & 0.15 & $311(62 \%)$ \\
3 & 0.25 & 0.2 & $369(74 \%)$ \\
\hline \hline
\end{tabular}

propagated impact points and the true impact points for the stable cases in each Monte Carlo simulation. In general, results shown in Table 2 demonstrate that higher launch perturbations lead to higher likelihood of instability, primarily because fast-mode angle of attack is larger when liquid resonance is encountered. Note that, regardless of the size of launch perturbations, all liquid instability occurs before apogee, with the vast majority occurring between 6 and $10 \mathrm{~s}$ into the trajectory. Projected impact point locations for unstable cases show that, although instability is induced early in flight, the impact locations are only approximately $4 \mathrm{~km}$, or about $25 \%$, short of the nominal impact location for stable trajectories. This is an interesting result that is characteristic of liquid instabilities observed in practice.

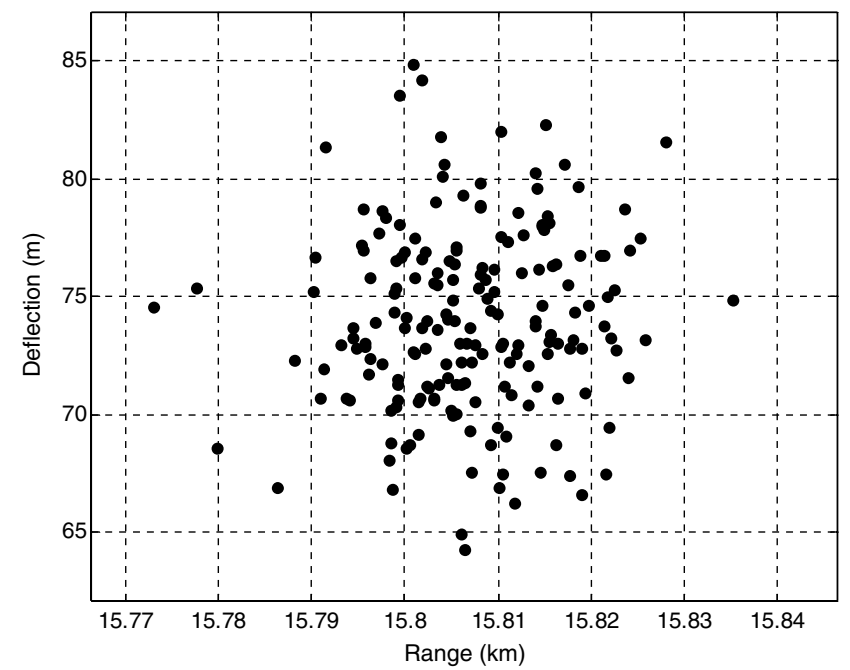

Fig. 19 Dispersion pattern for stable trajectories (Monte Carlo simulation 2,189 stable trajectories).

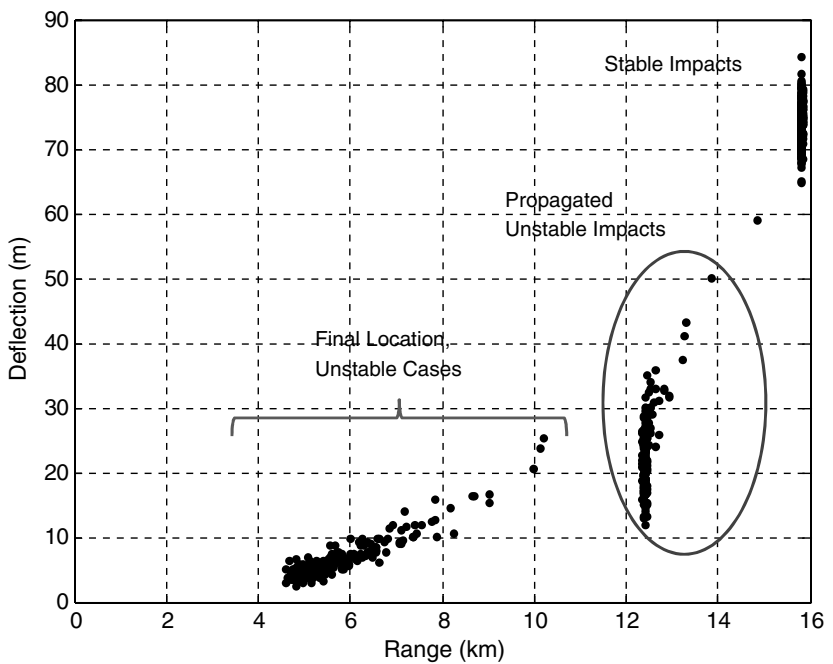

Fig. 20 Impact locations and locations of observed instability (Monte Carlo simulation 1).

\section{Parametric Trade Studies}

Although the previous Monte Carlo simulations provide insight into the effect of initial launch perturbations, they provide little guidance on how design factors might influence the likelihood of liquid instability occurring. Additional parametric trade studies were performed to evaluate the effect of liquid cavity aspect ratio and spinup time. Monte Carlo simulations were run with a variety of different viscous bearing coefficients $c_{v}$ and cavity aspect ratios AR, where $\mathrm{AR}$ is defined as the cylinder half-length divided by the radius. Table 3 lists the ranges of $c_{v}$ and AR that were considered. For each value of $c_{v}$ and $\mathrm{AR}$, a 500-trajectory Monte Carlo simulation was performed using the error budget of Monte Carlo simulation 2 in the previous section. In each Monte Carlo run, both the percentage of flights that exhibited instability and the mean time at which instability occurred were determined.

Figure 23 shows the percentage of flights exhibiting instability as a function of cavity aspect ratio and liquid viscous bearing coefficient. Note that several clear trends are present. The first is that, as AR increases, the percentage of flights exhibiting instability decreases. This is due to a shift in the nondimensional frequency $\tau$ at which liquid resonance peaks occur, which occur at larger $\tau$ for larger aspect ratios. As the liquid spins up to the projectile roll rate after launch, $\tau$ (defined as the natural frequency of the projectile nondimensionalized by the liquid spin rate) quickly decreases toward zero as

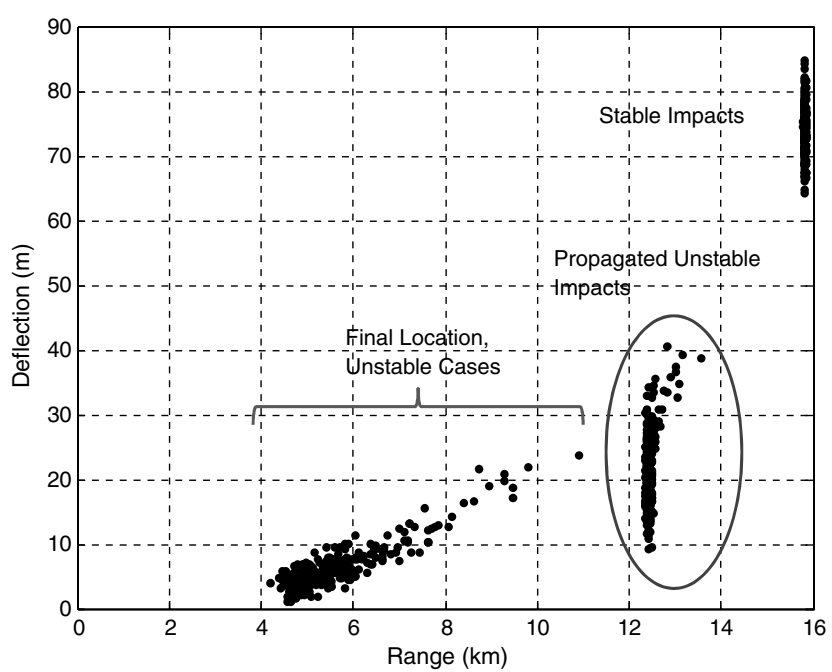

Fig. 21 Impact locations and locations of observed instability (Monte Carlo simulation 2).

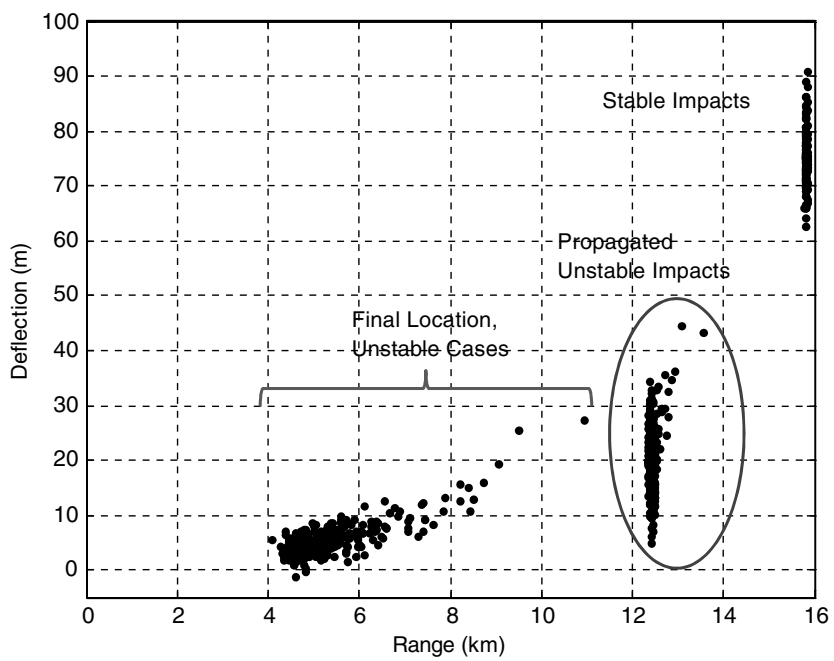

Fig. 22 Impact locations and locations of observed instability (Monte Carlo simulation 3) 
Table 3 Trade study parameter variations

\begin{tabular}{ll}
\hline \hline Parameter & \multicolumn{1}{c}{ Variation range } \\
\hline$c_{v}, \mathrm{~N} \cdot \mathrm{m}^{2} / \mathrm{s}$ & $0.0001-0.01$ (log scale) \\
$\mathrm{AR}$ & $1.118-4.1$ \\
\hline \hline
\end{tabular}

demonstrated in Fig. 13. Thus resonance dwell time, or the overall duration that the resonance is encountered during flight before the liquid spins up through it, is longer for smaller aspect ratios because the peaks occur closer to $\tau=0$. Naturally, longer dwell times at liquid resonance create a higher likelihood of instability because resonance amplifies angle of attack for a longer period.

The second trend evident from Fig. 23 is that higher viscous bearing coefficients (resulting in faster liquid spin-up times) lead to a higher percentage of unstable flights. Recall that a higher bearing coefficient means that the liquid spins up faster and encounters liquid resonance sooner after launch. Because liquid instability is caused by an unstable amplification of fast-mode angle of attack, encountering the resonance sooner in flight means that angle of attack perturbations created at launch have less time to dissipate and thus are larger when then liquid resonance is encountered. Note that, for very small viscous bearing coefficients (less than about $0.0002 \mathrm{~N} \cdot \mathrm{m}^{2} / \mathrm{s}$ ), liquid resonance is encountered so late in flight that all launch perturbations have dissipated, resulting in zero unstable cases observed. For viscous coefficients of less than about

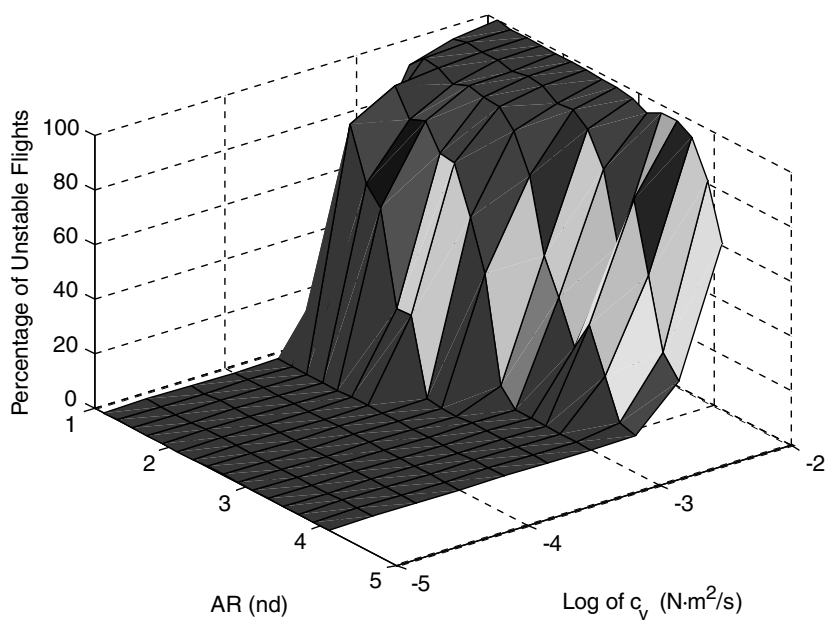

Fig. 23 Percentage of unstable flights vs AR vs $c_{v}$.

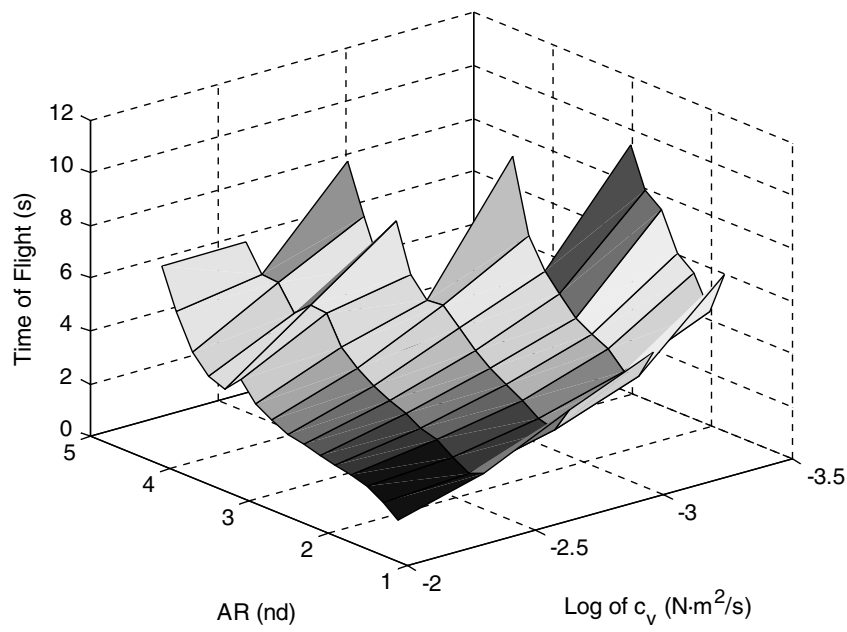

Fig. 24 Mean time of flight that instability detected vs AR vs $c_{v}$.
$0.0003 \mathrm{~N} \cdot \mathrm{m}^{2} / \mathrm{s}$, liquid resonance is not encountered at all during flight due to excessively slow liquid spin-up rates, and thus no instability is observed.

Figure 24 shows the mean time that instability is detected in each Monte Carlo simulation as a function of $\mathrm{AR}$ and $c_{v}$. The trends in Fig. 24 verify that instability occurs earlier in flight for larger viscous bearing coefficients because the projectile encounters liquid resonance sooner in flight, before launch perturbations have a chance to dissipate. However, the figure also shows an interesting trend with respect to aspect ratio. Resonances are encountered later in flight for smaller aspect ratios, and thus one might expect instability to be observed later in the trajectory. At the same time, however, dwell time for lower aspect ratios is significantly longer. Longer dwell times result in higher angle of attack perturbations input into the trajectory, which cause instability to occur almost immediately. Shorter dwell times, on the other hand, occurring for larger aspect ratios, generate smaller perturbations that take longer to cause dynamic instability due to their lower magnitude. For some trajectories, these small perturbations eventually build up as the projectile nears apogee, eventually leading to instability. Thus, Fig. 24 demonstrates the trend that larger aspect ratios cause instability later in flight even though resonance is encountered sooner. This is due to the lower overall perturbations caused by resonance with very low dwell times.

These parametric studies suggest certain guidelines that can be used during projectile design to avoid or mitigate liquid instability. Longer cavities reduce the likelihood of liquid-induced instability and postpone the time at which it occurs until later in flight. This is advantageous in that the overall range reduction caused by liquid instability, if it occurs at all, will be less than if the onset of instability had occurred earlier in flight. Furthermore, prolonging liquid spin-up times allows more time for fast-mode angle of attack caused by launch perturbations to dissipate by the time resonance is encountered, also reducing the chance that liquid resonance will lead to instability. Thus, at least for cylindrically shaped liquid cavities, care should be taken to avoid engaging the liquid, allowing it to spin up slowly so that launch perturbations can dampen out. These conclusions are obviously not valid for actively controlled projectiles in which control systems may excite the fast-mode angle of attack at later points in flight.

\section{Conclusions}

A novel liquid payload flight simulation tool has been described. The model uses a 7-degree-of-freedom dual-spin projectile model in which the liquid payload is considered to be a uniform body rolling separately from the projectile. Liquid moment coefficients are introduced by solving the linearized Navier-Stokes equations at steady-state flight conditions assuming quasi-static coning motion. A linear least-squares filter embedded within the simulation extracts fast- and slow-mode angles of attack at each time step, driving computations of liquid moments. Example results demonstrate that small initial launch perturbations can excite fast-mode angle of attack enough to initiate liquid instability. Both example results and Monte Carlo simulations demonstrate that liquid instability is characterized by positive feedback between fast-mode angle of attack and liquid payload moments, leading to flight instabilities occurring before apogee but resulting in range reductions of less than approximately $30 \%$. Monte Carlo simulations further verify that larger launch perturbations promote liquid instability by increasing fast-mode angle of attack when liquid resonance occurs. Trade studies analyzed the effects of cavity aspect ratio and liquid spin-up time, showing that cavities of higher aspect ratio and slower spin-up times serve to mitigate liquid instability.

\section{References}

[1] D'Amico, W. P., and Mark, A., "The Application of a Highly Permeable Medium to Reduce Spin-Up Time and to Stabilize a Liquid-Filled Shell," U.S. Army Ballistic Research Lab., Rept. MR-02851, Aberdeen Proving Ground, MD, July 1978.

[2] D'Amico, W. P., and Clay, W. H., "Flight Tests for Prototype Felt Wedge/White Phosphorous Improved Smoke Concept," U.S. Army 
Ballistic Research Lab., Rept. MR-02824, Aberdeen Proving Ground, MD, April 1978

[3] Cooper, G. R., "Moment Exerted on a Coning Projectile by a Spinning Liquid in a Cylindrical Cavity Containing a Porous Medium," U.S. Army Ballistic Research Lab., Rept. MR-3677, Aberdeen Proving Ground, MD, June, 1988.

[4] Cooper, G. R., "Spinning Projectile with an Inviscid Liquid Payload Impregnating Porous Media," AIAA Journal, Vol. 46, No. 3, March 2008, pp. 783-787. doi:10.2514/1.30481

[5] Cooper, and Gene, R., "Moments on a Coning M864 by a Liquid Payload: The Candlestick Problem and Porous Media," U.S. Army Research Lab., TR 3837, Aberdeen Proving Ground, MD, July 2006.

[6] Dean, C., Weber, D., Molnar, J., Hollis, M., D'Amico, W., Brandon, F., et al., "Savage Medical Resupply Projectile," U.S. Army Armament Munition, Rept. A808233, Aberdeen Proving Ground, MD, Oct. 1996.

[7] Stewartson, K., "On the Stability of a Spinning Top Containing Liquid," Journal of Fluid Mechanics, Vol. 5, No. 4, 1959, pp. 577-592. doi:10.1017/S0022112059000404

[8] Karpov, B. G., "Experimental Observation of the Dynamic Behavior of Liquid Filled Shell,' U.S. Army Ballistics Research Lab., Rept. 1171, Aberdeen Proving Ground, MD, Aug. 1961.

[9] Mark, A., and Mermagen, W. H., "Measurement of Spin Decay and Instability of Liquid-Filled Projectiles via Telemetry," U.S. Army Ballistics Research Lab., Rept. 2333, Aberdeen Proving Ground, MD, Oct. 1973.

[10] Kitchens, C., Jr., Gerber, N., and Sedney, R., "Spin Decay of LiquidFilled Projectiles," Journal of Spacecraft and Rockets, Vol. 15, Nov.Dec. 1978 , pp. $348-354$ doi: $10.2514 / 3.57326$

[11] Sedney, R., "A Survey of the Fluid Dynamic Aspects of Liquid-Filled Projectiles," 12th AIAA Atmospheric Flight Mechanics Conf., Snowmass, CO, AIAA Paper 1985-1822, Aug. 1985.

[12] Wedemeyer, E. H., "Viscous Corrections to Stewartson's Stability Criterion," U.S. Army Ballistics Research Lab., Rept. 1325, Aberdeen Proving Ground, MD, June 1966.

[13] Kitchens, C. W., Jr., Gerber, N., and Sedney, R., "Oscillations of a Liquid in a Rotating Cylinder: Part 1. Solid-Body Rotation,” U.S. Army Ballistics Research Lab., TR 02081, June 1978.

[14] Frasier, J. T., and Scott, W. E., "Stability of a Liquid-Filled Gyroscope: Inviscid Analysis, Viscous Correction, and Experiments," Journal of Spacecraft and Rockets, Vol. 8, No. 5, May 1971, pp. 523-526. doi: $10.2514 / 3.30302$

[15] Weber, D. J., "Simplified Method for Evaluating the Flight Stability of Liquid-Filled Projectiles," Journal of Spacecraft and Rockets, Vol. 31, No. 1, 1994, pp. 130-134. doi: $10.2514 / 3.26412$

[16] Vaughn, H. R., Wolfe, W. P., and Oberkampf, W. L., "Flight Trajectory Simulation of Fluid Payload Projectiles," Journal of Guidance, Control, and Dynamics, Vol. 9, No. 2, March-April 1986, pp. 213-220. doi: $10.2514 / 3.20092$

[17] Cooper, G., and Costello, M., "Trajectory Predictions of Spin-
Stabilized Projectiles with a Liquid Payload," Journal of Spacecraft and Rockets, Vol. 48, No. 4, July-Aug. 2011, pp. 664-670. doi: $10.2514 / 1.52564$

[18] Abramson, N. H., "Dynamics of Contained Liquids: A Personal Odyssey," Applied Mechanics Reviews, Vol. 56, No. 1, Jan. 2003, pp. R1-R7. doi:10.1115/1.1511517

[19] Pocha, J. J., "An Experimental Investigation of Spacecraft Sloshing," Space Communication and Broadcasting, Vol. 5, No. 4, 1987, pp. 323 332.

[20] Agrawal, B. N., "Dynamic Characteristics of Liquid Motion in Partially Filled Tanks of a Spinning Spacecraft," Journal of Guidance, Control, and Dynamics, Vol. 16, No. 4, July-Aug. 1993, pp. 636-640. doi: $10.2514 / 3.21061$

[21] Manasseh, R., "Visualization of the Flows in Precessing Tanks with Internal Baffles," AIAA Journal, Vol. 31, No. 2, Feb. 1993, pp. 312-318. doi:10.2514/3.11669

[22] Deffenbaugh, D., Dodge, F., and Green, S., "Final Report for the Liquid Motion in a Rotating Tank Experiment (LME)," NASA CR 1998208667, Sept. 1998

[23] Kobine, J. J., "Inertial Wave Dynamics in a Rotating and Precessing Cylinder," Journal of Fluid Mechanics, Vol. 303, 1995, pp. 233-252. doi:10.1017/S0022112095004253

[24] Bao, G. W., and Pascal, M., "Stability of a Spinning Liquid-Filled Spacecraft," Archive of Applied Mechanics, Vol. 67, 1997, pp. 407-421. doi:10.1007/s004190050127

[25] Gerrits, J., and Veldman, A., "Dynamics of Liquid-Filled Spacecraft," Journal of Engineering Mathematics, Vol. 45, 2003, pp. 21-38. doi:10.1023/A:1022055916067

[26] Costello, M., and Peterson, A., "Linear Theory of a Dual-Spin Projectile in Atmospheric Flight," Journal of Guidance, Control, and Dynamics, Vol. 23, No. 5, Sept.-Oct. 2000, pp. 789-797. doi: $10.2514 / 2.4639$

[27] Murphy, C. H., "Angular Motion of a Spinning Projectile with a Viscous Liquid Payload," Journal of Guidance, Control, and Dynamics, Vol. 6 , No. 4, July-Aug. 1983 , pp. 280-286. doi: $10.2514 / 3.19830$

[28] Murphy, C. H., "A Relation Between Liquid Roll Moment and Liquid Side Moment," Journal of Guidance, Control, and Dynamics, Vol. 8 , No. 2, March-April 1985, pp. 287-288. doi:10.2514/3.19977

[29] Murphy, C. H., Bradley, J. W., and Mermagen, W. H., "Side Moment Exerted by a Spinning Coning Highly Viscous Liquid Payload," U.S. Army Ballistics Research Lab., TR 3074, Aberdeen Proving Ground, MD, Dec. 1989.

[30] Murphy, C. H., "Free Flight Motion of Symmetric Missiles," U.S. Army Ballistics Research Lab., Rept. 1216, Aberdeen Proving Ground, MD, July 1963.
D. Spencer

Associate Editor 